\title{
Big Bang Blackbody Simulator
}

\author{
Sara Stanchfield \\ University of Wisconsin - Madison
}

\begin{abstract}
The Cosmic Microwave Background (CMB) is a remnant glow from the Big Bang, and as such it provides us with a direct view into the early universe. By mapping out the CMB we are able to view the universe as it appeared when it was only 400,000 years old. The CMB radiates as an almost perfect blackbody at a temperature of 2.7 Kelvin. It is necessary to be able to simulate the $\mathrm{CMB}$ as a blackbody source in order to allow us to test instrumentation intended for $\mathrm{CMB}$ observation. The purpose of the Big Bang Blackbody Simulator is to construct a blackbody 'cold load' to measure the microwave response of superconducting Transition-Edge Hot-Electron Microbolometers. These detectors will allow us to measure the faint polarization signals in the CMB that are expected to be the result of gravitational waves generated in the very first moments of the universe.
\end{abstract}

\section{Introduction}

Around 400,000 years after the Big Bang, during an era termed 'recombination' the universe had cooled to a point where the photons could no longer scatter with the surrounding baryons. These photons were then allowed to travel freely through space and are what we observe as the Cosmic Microwave Background (CMB) today. The CMB radiates as a nearly perfect blackbody at 2.7 Kelvin. The CMB also contains very faint temperature and polarization inhomogeneities. Around $10^{-34}$ seconds after the Big Bang a period of rapid expansion occurred. This rapid expansion period, called inflation, produced gravitational waves which would have left an imprint on the polarization of the CMB.

The focus of the Big Bang Blackbody Simulator (BBS) is to create a calibrated source of microwave photons that simulate the $\mathrm{CMB}$ as observed from space or the ground in order to measure the response of superconducting the Transition-Edge Hot-Electron Microbolometer (THM). These THM detectors are specifically optimized to take measurements of the CMB. Arrays of 1000's of THM detectors would be sensitive enough to measure the very faint B-mode polarizations in the $\mathrm{CMB}$, the imprint left by gravitational waves.

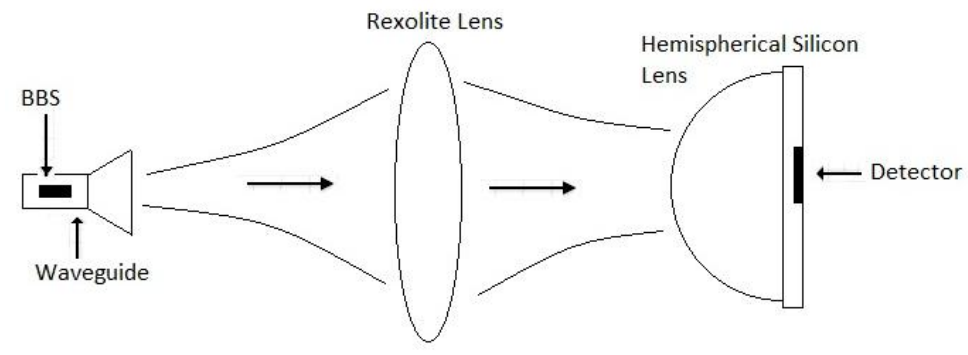

Figure 1: The BBS radiation will be coupled to the THM detector through multiple lenses.

\section{Acknowledgements:}

Special thanks to Peter Timbie, Emily Barrentine, and Eric Katzelnick, also to Wisconsin Space Grant Consortium for financial support. 
Figure 1 shows how the BBS will be used to test the THM detector. The radiation produced by the BBS will be coupled to the detector through a series of lenses. This entire set up will be then be placed in a cryostat with the left side being cooled to 4 Kelvin and the right to approximately $100 \mathrm{mKelvin}$ to reduce noise.

\section{Construction}

The BBS consists primarily of a 100 Ohm chip resistor and a copper finline structure, shown in figure 2 . When heated, the chip resistor emits thermal radiation which is then coupled into a copper waveguide by the microwave circuit created by the copper finline structure. The copper finline structure is the placed between two pieces of Kapton to electrically and thermally isolate the structure as well as provide mechanical stability.

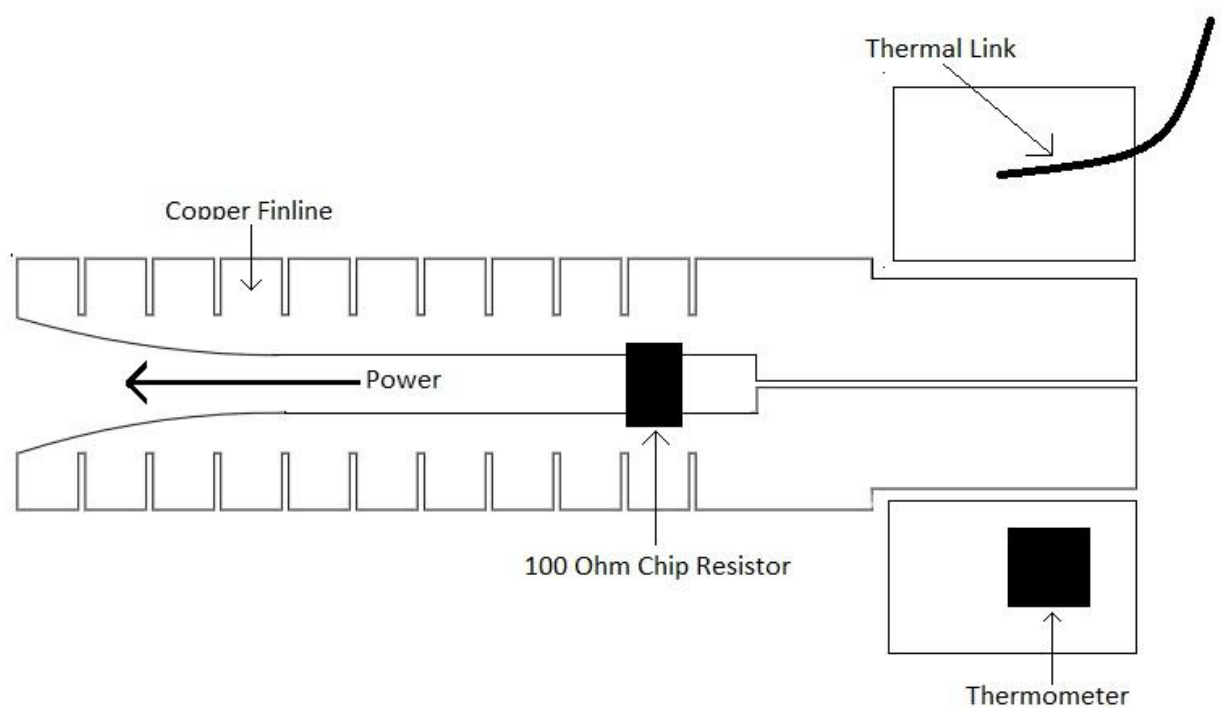

Figure 2: The structure of the BBS.

A Ruthenium Oxide thermistor is used to monitor the temperature of the BBS. A thick copper wire also acts as a thermal think between the BBS and the cold stage, which is used to control how quickly the BBS can be heated and cooled.

\section{Requirements of the Big Bang Blackbody Simulator}

In order for the BBS to be effective it must meet a few basic requirements. The first is that it simulates an almost perfect blackbody with an emissivity of $90 \%$ or greater. It also needs to emit at the peak of the CMB spectrum, 75 to $110 \mathrm{GHz}$ or approximately $3 \mathrm{~mm}$. Next we must be able to heat the BBS to the desired temperature using low power, 10 mWatts or less. The desired temperature for CMB simulation is 4 to 20 Kelvin. This process of heating and cooling must also happen very quickly in order to viable for laboratory experiments, on the order of one second. To achieve this, the BBS must have low heat capacity and an optimal thermal conductance. 


\section{Results}

\section{Emissivity}

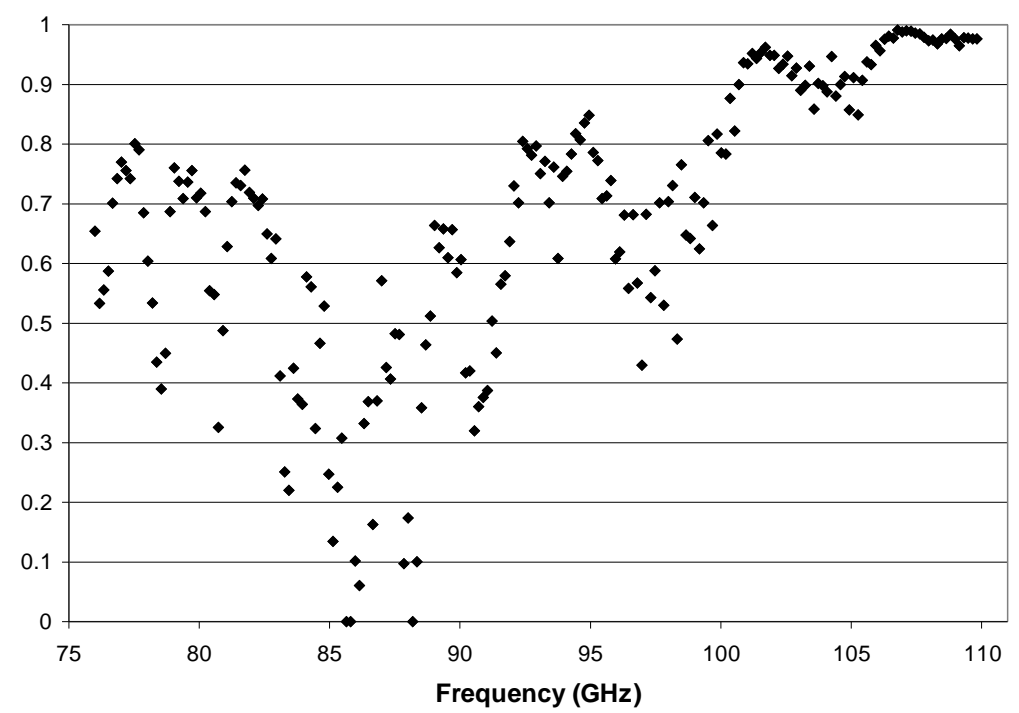

Figure 3: Plot of the emissivity of the BBS versus frequency

Figure 2 shows the emissivity of the BBS as a function of frequency. The emissivity of the BBS peaks around $98 \%$ with average emissivity of $69 \%$, which is near the desired level.

To measure the time constant of the BBS, the BBS was placed in a copper waveguide block and then attached to the cold stage in a cryostat cooled with liquid helium to 4 Kelvin. The BBS was then heated to a maximum of 20 Kelvin using Joule power. Figure 4 shows the rise and fall of the temperature of the BBS. Joule power was applied until the temperature stabilized, then the power was shut off and the BBS was allowed to cool.

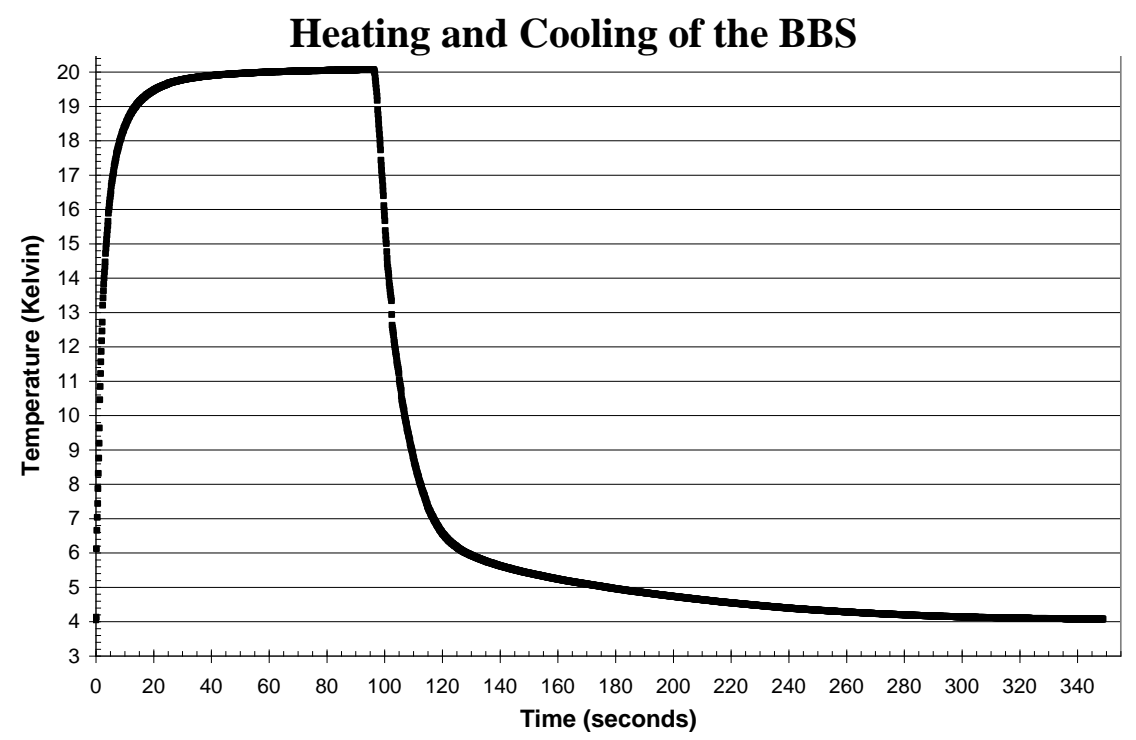

Figure 3: The BBS reaches a maximum temperature of 20 Kelvin when 34 mWatts power applied. 
Fitting the temperature versus time curve to an exponential gives a time constant of 3.3 seconds. Varying amounts of power were applied to the BBS to reach maximum temperatures between 12 and 20 Kelvin. Using the power versus temperature graph, figure 4 , the thermal conductance, $\mathrm{G}$, was determined by fitting the line to the equation

Then,

$$
\mathrm{P}=\mathrm{C}^{*}\left(\mathrm{~T}^{\mathrm{n}}-4^{\mathrm{n}}\right)
$$

$$
\mathrm{G}={ }^{\mathrm{dP}} / \mathrm{dT}=\mathrm{n} * \mathrm{C} * \mathrm{~T}^{(\mathrm{n}-1)}
$$

Using these equations, the thermal conductance of the BBS was found to be $2.9 \times 10^{-3} \mathrm{~W} / \mathrm{K}$. The heat capacity, the product of the time constant and thermal conductance, was determined to be $9.7 \times 10^{-3} \mathrm{~J} / \mathrm{K}$.

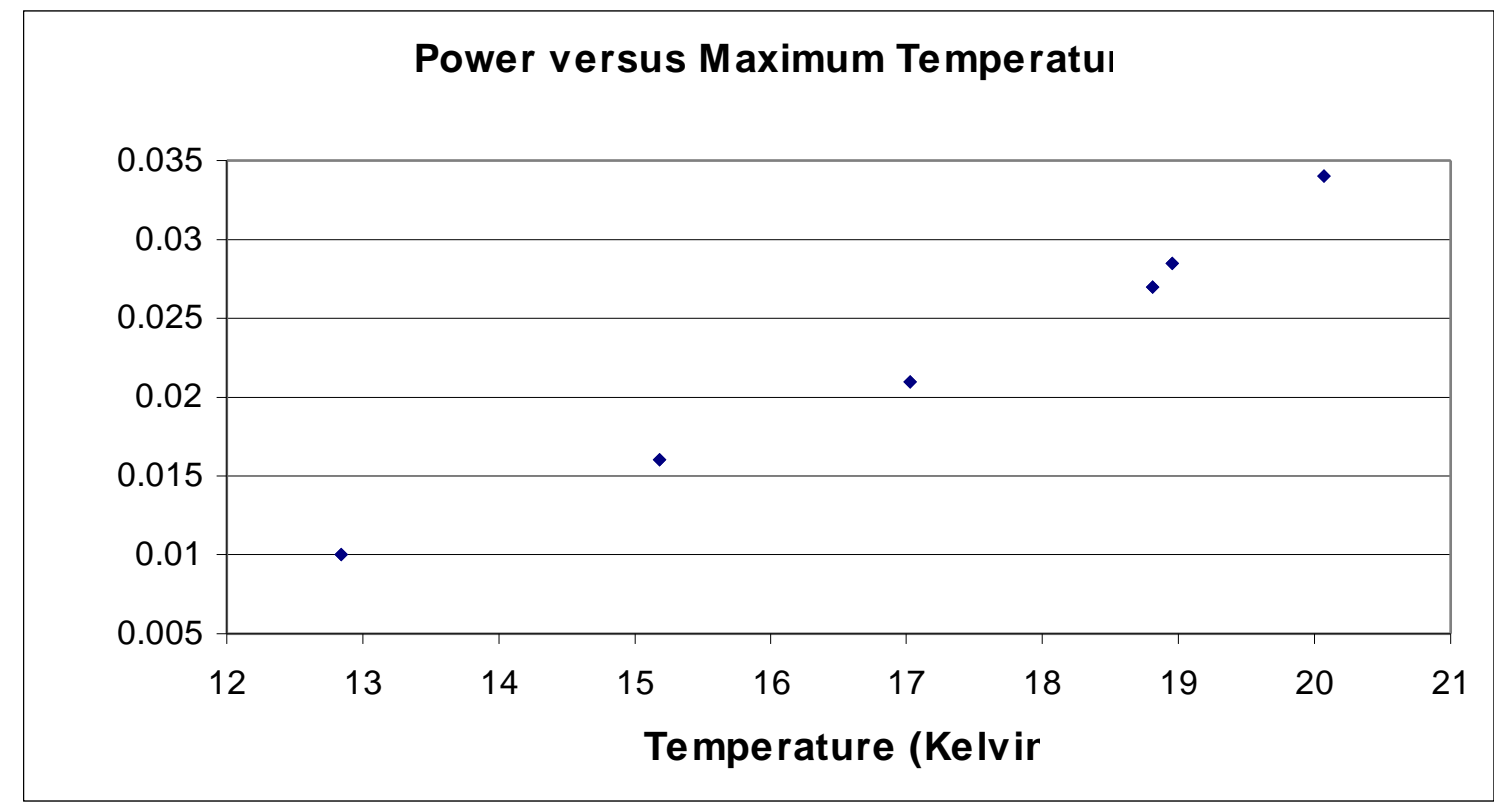

Figure 4: Plotting the power needed to heat the BBS to each maximum temperature gives a thermal conductance of $2.9 \times 10^{-3} \mathrm{~W} / \mathrm{K}$.

\section{Conclusions}

The initial thermal requirements placed on the BBS, a short time constant and low power usage have been satisfied. The time constant to heat or cool the BBS is approximately 3 seconds, and a maximum of $35 \mathrm{mWatts}$ of power was used, both of which are in the desired range. While the emissivity of the BBS is reasonable, there is some room for improvement. Future work will use two chip resistors in the place of the current one, as preliminary simulations have shown higher emissivity when this resistive area is better distributed in the finline structure.

Next, the Big Bang Blackbody Simulator will be used to test the microwave sensitivity of Transition-Edge Hot-Electron Microbolometer detectors. If successful, these detectors may be used in NASA's CMBPol mission at the end of the decade. 